\title{
Biological Maternal Grandfather
}

National Cancer Institute

\section{Source}

National Cancer Institute. Biological Maternal Grandfather. NCI Thesaurus. Code C96577.

A male relative who is the biological father of the biological mother. 